Document downloaded from:

http://hdl.handle.net/10251/159988

This paper must be cited as:

Moliner, L.; Ilisie, V.; González Martínez, AJ.; Oliver-Gil, S.; Gonzalez, A.; Giménez-

Alventosa, V.; Cañizares, G.... (2019). TOF studies for dedicated PET with open geometries. Journal of Instrumentation. 14:1-8. https://doi.org/10.1088/1748-0221/14/02/C02006

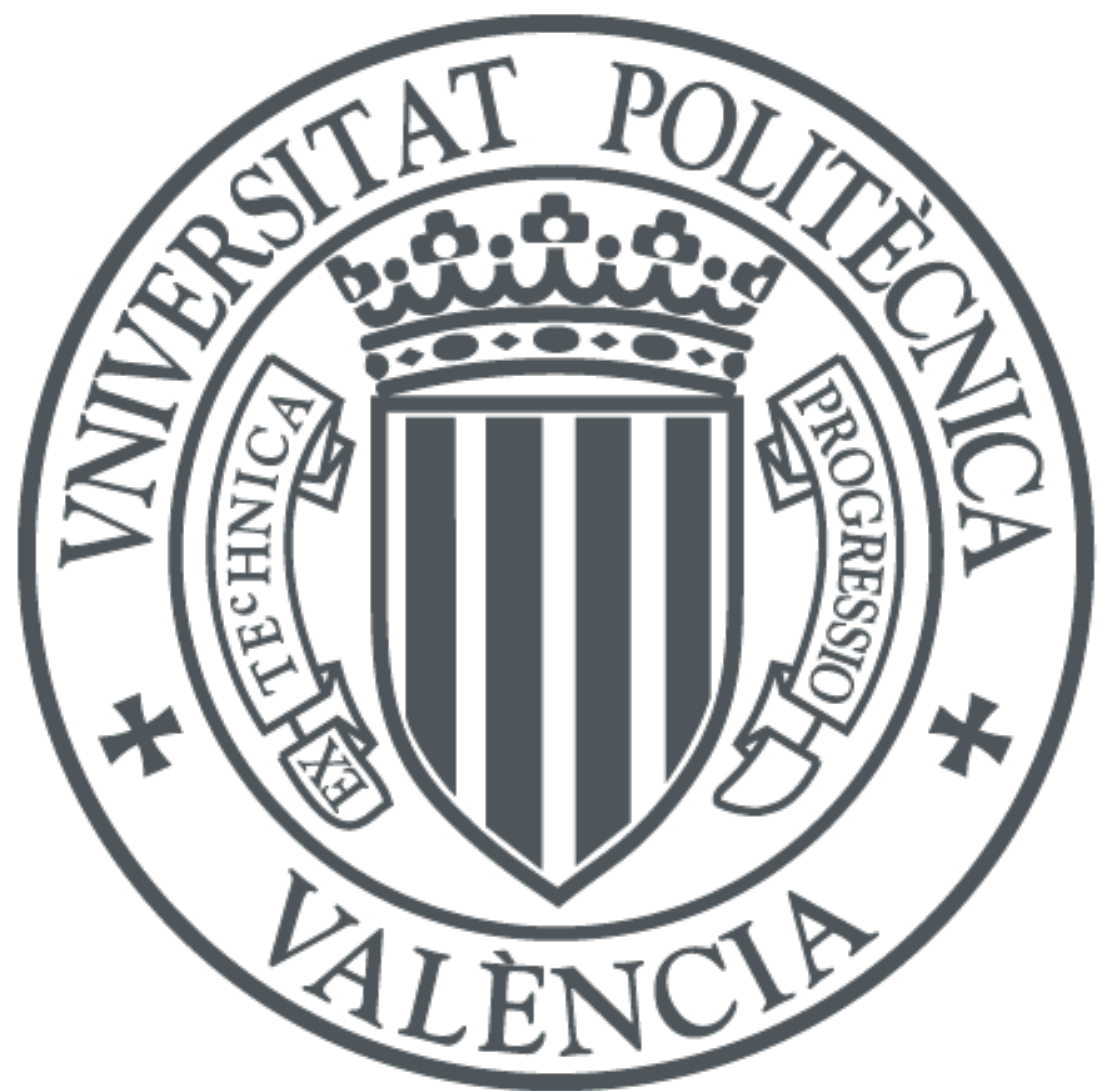

The final publication is available at

https://doi.org/10.1088/1748-0221/14/02/C02006

Copyright IOP Publishing

Additional Information 


\title{
TOF studies for dedicated PET with open geometries
}

\author{
Laura Moliner, ${ }^{\mathrm{a},{ }^{*}}$ Victor Ilisie ${ }^{\mathrm{a}}$, Antonio Gonzalez ${ }^{\mathrm{a}}$, Sandra Oliver ${ }^{\mathrm{a}}$, Andrea \\ Gonzalez ${ }^{\mathrm{a}}$, Vicent Gimenez-Alventosa ${ }^{a}$, Gabriel Cañizares ${ }^{\mathrm{a}}$, Efthimis Lamprou ${ }^{\mathrm{a}}$, \\ Jorge Alamo ${ }^{b}$, Filomeno Sanchez ${ }^{\mathrm{a}}$, Maria Jose Rodriguez ${ }^{\mathrm{a}}$ and Jose M. Benlloch ${ }^{\mathrm{a}}$ \\ ${ }^{a}$ i3M, Institute for Instrumentation in Molecular Imaging, Universitat Politècnica de València - CSIC, \\ Cami de Vera $s / n$, Valencia, Spain, \\ ${ }^{b}$ Oncovision, Jeronimo Monsoliu 92, Valencia, Spain \\ E-mail: lmoliner@i3m.upv.es
}

\begin{abstract}
:
Recently, two novel PET devices have been developed with open geometries, namely: breast and prostate-dedicated scanners. The breast-dedicated system comprises two detector rings of twelve modules with a field of view of $170 \mathrm{~mm}$ x $170 \mathrm{~mm}$ x $94 \mathrm{~mm}$. Each module consists of a continuous trapezoidal LYSO crystal and a PSPMT. The system has the capability to vary the opening of the rings up to $60 \mathrm{~mm}$ in order to allow the insertion of a needle to perform a biopsy procedure. The prostate system has an open geometry consisting on two parallel plates separated $28 \mathrm{~cm}$. One panel includes 18 detectors organized in a $6 \times 3$ matrix while the second one comprises 6 detectors organized in a $3 \times 2$ matrix. All detectors are formed by continuous LYSO crystals of $50 \mathrm{~mm} \times$ $50 \mathrm{~mm} \times 15 \mathrm{~mm}$, and a SiPM array of $12 \times 12$ individual photo-detectors. The system geometry is asymmetric maximizing the sensitivity of the system at the prostate location, located at about $2 / 3$ in the abdomen-anus distance.
\end{abstract}

The reconstructed images for PET scanners with open geometries present severe artifacts due to this peculiarity. These artifacts can be minimized using Time Of Flight information (TOF). In this work we present a TOF resolution study for open geometries. With this aim, the dedicated breast and prostate systems have been simulated using GATE (8.1 version) with different TOF resolutions in order to determine the image quality improvements that can be achieved with the existing TOF-dedicated electronics currently present in the market. The images have been reconstructed using the LMOS algorithm including TOF modeling in the calculation of the voxelLine Of Response emission probabilities.

KEYWORDS: PET; dedicated breast PET; breast cancer; breast biopsy system.

${ }^{*}$ Corresponding author. 


\section{Contents}

Contents $\quad 2$

1. Introduction $\quad 2$

2. Materials and methods $\quad 3$

2.1 MAMMOCARE system 3

2.2 PROSPET SYSTEM 4

2.3 Methods 4

4. Summary and conclusions $\quad 6$

$\begin{array}{lr}\text { Acknowledgments } & 6\end{array}$

$\begin{array}{lr}\text { References } & 7\end{array}$

\section{Introduction}

Currently, there are three trends in the design of human Positron Emission Tomograph (PET) scanners. The conventional one is roughly based on the design of detector rings of about $70 \mathrm{~cm}$ of transaxial Field Of View (FOV) and about $20 \mathrm{~cm}$ of axial FOV. A second approach in the research area consists in the design of total body PET systems that aim to completely explore the patient. It is essentially characterized by a $200 \mathrm{~cm}$ axial length [1]. The last approach consists in the design of less-bulky and cost-effective, organ-dedicated systems [2-6]. The development of organ-dedicated PET systems tries to overcome current limitations in prevention, diagnosis and treatment of certain common diseases. These systems can have certain advantages such as, enhancement of sensitivity, increasing the solid angle of detection, improving the spatial resolution to provide more detail of the structure of pathologies, lowering the tracer dose, reducing the scanner costs, even providing portability due to their reduced dimensions or a combination of them.

Cylindrical PET geometries (with axial symmetry) provide an optimal performance and sensitivity. However, the most suitable geometries for dedicated PET scanners exhibit a limited angular tomography that varies depending on the application. This is a serious drawback, as the sensitivity is drastically reduced. Besides there is a lack of information about the opening axis, which causes serious artifacts. One way to partially remove artifacts is by using the Time Of Flight (TOF) information of the annihilation photons in the image reconstruction process. This consists in measuring the time difference between arrival of the two photons and localizing the position of the positron annihilation along the Line Of Response (LOR). The accuracy of the method will dependent on the time measurement precision. With this method one can reduce the uncertainty of the emission probability of the voxels for the corresponding LORs (see figure 1).

Our group has a large experience working in the development of dedicated PET systems. Recently, two PET scanners have been developed with open geometries: breast and prostatededicated systems. In this work we present a TOF resolution study for such open geometries. In order to perform our analysis, these two systems have been simulated with GATE 8.1 version (http://www.opengatecollaboration.org) using different TOF resolutions to determine the image quality improvements that can potentially be obtained with the existing TOF-dedicated electronics present in the market. The images have been reconstructed using a modified TOR 
projector [7] with List Mode algorithm [8], which includes the TOF modeling in the calculation of voxel-LOR emission probabilities.

A)

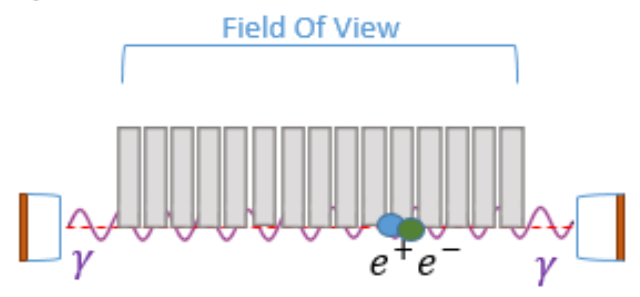

B)

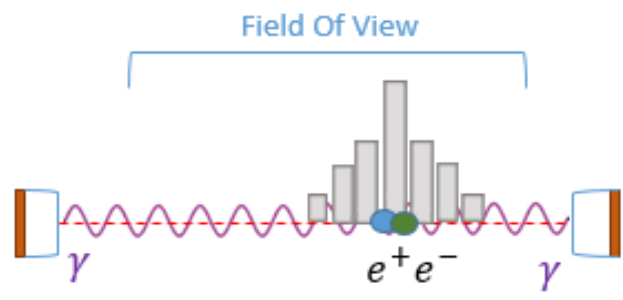

Figure 1. TOF schematics. A) Without TOF information, all the voxels in the LOR have the same emission probability $B)$ with TOF information, the emission probability is modeled according to the accuracy of time.

\section{Materials and methods}

\subsection{MAMMOCARE system}

Mammography is the standard imaging technique for breast scanning. This scanning is crucial in order to recognize a disease in early stages, however it is not enough to assess whether a suspicious finding represents breast cancer, another type of pathology, or just normal tissue. Less than $1 \%$ of the patients with suspicious results do actually have cancer, therefore, most of abnormal mammograms are false-positives requiring an additional evaluation [9]. Last trends in research for breast cancer diagnosis have demonstrated the potential of dedicated Breast Positron Emission Tomography (dBPET) functional imaging as a complementary diagnosis tool and it is specially indicated for women for whom evaluation and diagnosis with standard methods present additional difficulties (i.e., cystic or radiographically dense breast) [10].

The MAMMOCARE project has developed a dBPET-guided breast biopsy system [11] (see figure 2). The prototype consists of an examination table (where the patient is placed in prone position), a PET imaging module and complete biopsy system. The PET system comprises two detector rings of twelve modules each, with a field of view of $170 \mathrm{~mm} \times 170 \mathrm{~mm} \times 94 \mathrm{~mm}$. Each module contains a single LYSO continuous (not pixelated) scintillation crystal coupled to a PSPMT and electronic readout board. The system has the capability to vary the ring openings up to $60 \mathrm{~mm}$ in order to allow the insertion of a needle to perform the biopsy procedure. Two pallets support the breast during the biopsy procedure with a minimum compression. The soft compression is performed manually by the operator and includes a torque limiter to prevent possible patient injuries caused by the operator. One of the pallets presents an entrance window to allow the insertion of the needle. The system comprises two detector rings of twelve modules with a field of view of $170 \mathrm{~mm} \times 170 \mathrm{~mm} \times 94 \mathrm{~mm}$. 


\subsection{PROSPET system}

Prostate Cancer $(\mathrm{PCa})$ is the most common form of cancer in adult male, closely followed by lung and colorectal cancer. Furthermore, $\mathrm{PCa}$ is currently the second most common cause of cancer that causes death in adult male population. The most commonly used method for imaging the prostate is Trans Rectal Ultrasounds (TRUS) [12]. However, less than 60\% of tumors - usually advanced tumors - are visible with TRUS [13].

Our group is currently developing a dedicated prostate PET, PROSPET, with the aim of localizing this disease in early stages (see figure 3). This system presents an open geometry consisting on two parallel plates separated $340 \mathrm{~mm}$. One panel includes 18 detectors organized in a $6 \times 3$ matrix while the second one comprises 6 detectors organized in a $3 \times 2$ matrix. All detectors are formed by continuous LYSO crystals of $50 \mathrm{~mm} \times 50 \mathrm{~mm} \times 15 \mathrm{~mm}$ and a SiPM array of $12 \times 12$ individual photo-detectors. The system geometry is asymmetric maximizing the sensitivity of the system at the prostate location, at about $2 / 3$ from the abdomen-anus distance. The particular position of the patient (see figure 3) allows for the future addition of a biopsy system.
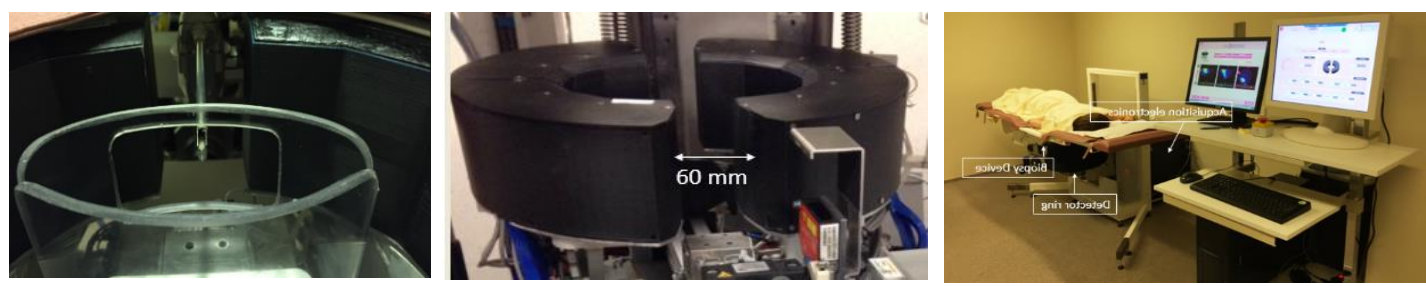

Figure 2. Left: detail of how the biopsy needle is inserted inside the dBPET. As can be seen, the ring opens and there is a pallet with a window through which the needle is inserted. Center: Mammocare detector when the configuration is totally opened. Right: real installation of Mammocare in NKI hospital (Amsterdam)
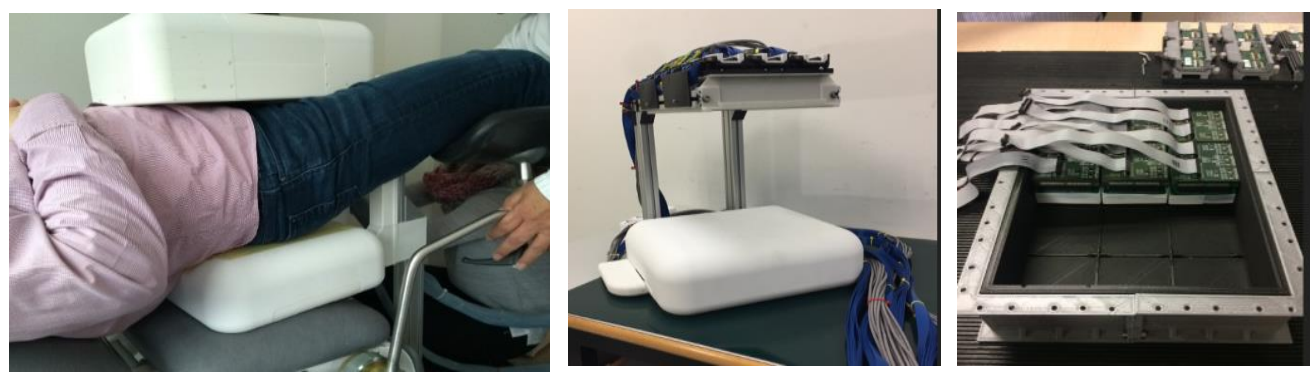

Figure 3. Left: patient positioning in PROSPET device. Center: detail of the two detector plates of PROSPET. Right: detail of the electronics of PROSPET, showing the LYSO crystal coupled to the SiPM and the front-end electronics.

\subsection{Methods}

In this work we evaluate the needed TOF resolution in order to eliminate the artifacts caused by the open geometry of the previously mentioned dedicated systems. With this aim, both systems have been simulated using GATE version 8.1 (see figure 4). Different virtual sources of $511 \mathrm{keV}$ back-to-back photons were simulated in order to accelerate the simulations. Each simulation lasted 5 seconds. Low statistics for the coincidences were recorded (approximately, 17M for MAMMOCARE and 20M for PROSPET), which were similar to a real acquisition. These sources were specifically defined and located in the FOV, in order to observe the artifacts produced by 
the open geometries. For MAMMOCARE, five spherical sources of $10 \mathrm{~mm}$ diameter have been simulated along its aperture axis. The spheres had $30 \mathrm{~mm}$ center -to -center distance. For PROSPET the simulation consisted of a sphere of $40 \mathrm{~mm}$ of diameter placed at the center of transaxial and axial FOV. The results of the simulations were reconstructed using different TOF resolutions, more specifically from 10 to 1000 ps for PROSPET and from 1000 to 200 ps for MAMMOCARE. The low TOF values analyzed for PROSPET system are not reachable with current technology, but this study can be regarded as a proof of concept in the image reconstruction field, for such open geometries. The used reconstruction algorithm is LMOS [8] with 2 iterations and 5 subsets. The voxel sizes for the reconstruction are $1 \mathrm{~mm} \times 1 \mathrm{~mm} \times 1 \mathrm{~mm}$ and with $2 \mathrm{~mm} \times 2 \mathrm{~mm}$ virtual pixel sizes to define the LORs. The resolution of the sources for MAMMOCARE and the Signal to Noise Ratio (SNR) for PROSPET were evaluated in the reconstructed images. To obtain the resolution, the FHWM of the sources was measured along the plane affected by the open geometry. To obtain the SNR we considered a spherical volume of interest of $30 \mathrm{~mm}$ of diameter inside a homogenous source in the reconstructed image, and, a volume of interest of $30 \mathrm{~mm}$ outside the source. The ratio between these two values is the calculated SNR.
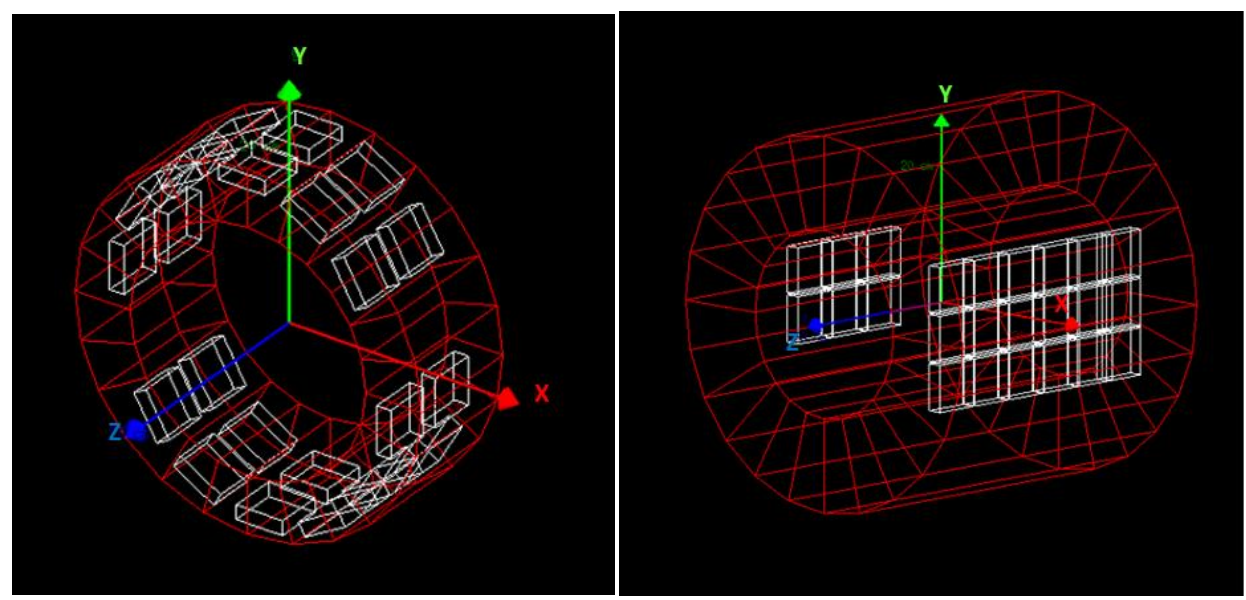

Figure 4. Sketch of the MAMMOCARE with open geometry (left) and of PROSPET (right) modelled in GATE.

\section{Results}

For MAMMOCARE system, the limited aperture of the system ring generates artefacts close to the border of the FOV, degrading the resolution of the sources. However, when TOF information is included, these artefacts tend to be reduced (see figure 5). We plot the values of the FWHM of the reconstructed sources versus TOF resolution. As we can see in figure 6, the results show an improvement in the resolution that reach reasonable values at 200ps.

Regarding the PROSPET system, the extremely open geometry of the system creates very severe artefacts in the reconstruction images without TOF. Figure 7 shows the reconstructed simulated cylinder without TOF and with different time resolutions. In figure 8 the logarithm of the SNR is represented versus time resolution. As we can see, increasing TOF resolution reduces the artefact effects, reaching a plateau for the SNR below $100 \mathrm{ps}$. 


\section{Summary and conclusions}

The state-of-the art commercial TOF-dedicated electronics reach temporal resolutions in the range of 300-400ps [14] and continue to improve. Experimental systems have reached up to 150 ps with only two detector blocks [15]. According to this simulation study the necessary TOF resolution needed to limit the artifacts generated by the open geometries for the simulated systems is of the order of 100ps for PROSPET and 200ps for MAMMOCARE.

\section{Acknowledgments}

This work was supported in part by the Spanish Government Grants TEC2016-79884-C2 and RTC-2016-5186-1 and by the European Research Council (ERC) under the European Union's Horizon 2020 research and innovation program (Grant Agreement No. 695536).
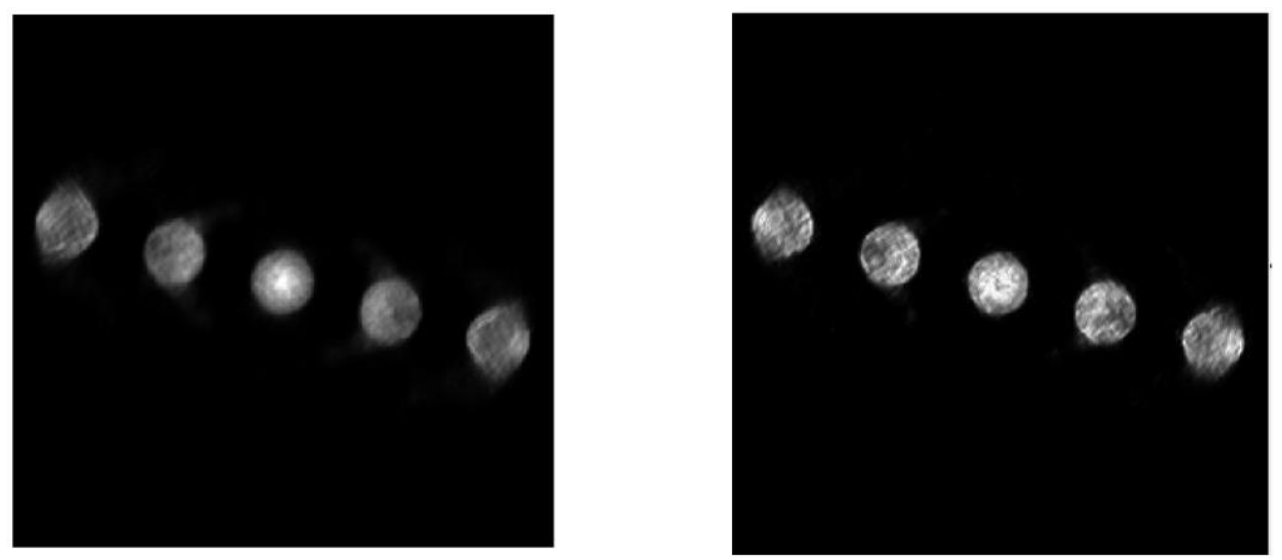

Figure 5. Left: reconstructed sources without TOF. Right: reconstructed sources with TOF with a resolution blurring of $200 \mathrm{ps}$.
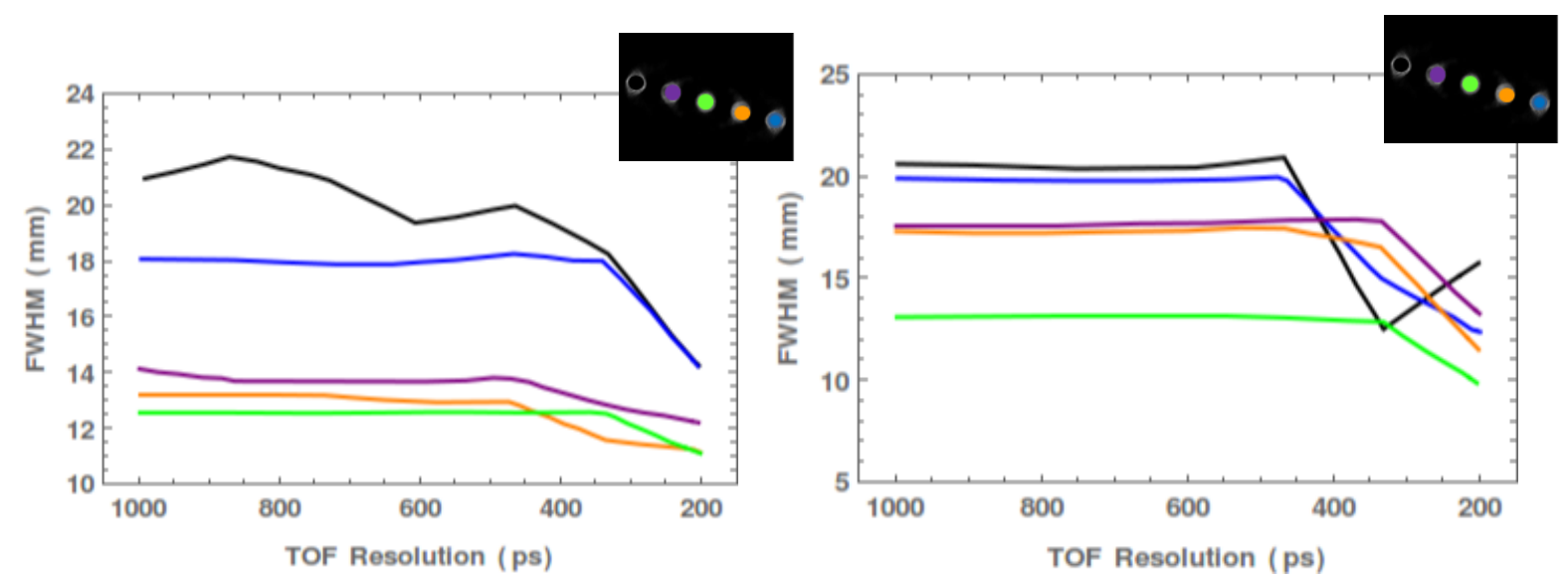

Figure 6. FWHM for reconstructed sources of MAMMOCARE system. Left: along of the aperture axis. Right: along the perpendicular axis to the aperture axis. Colours of the curves correspond to the image reconstructed legend. 


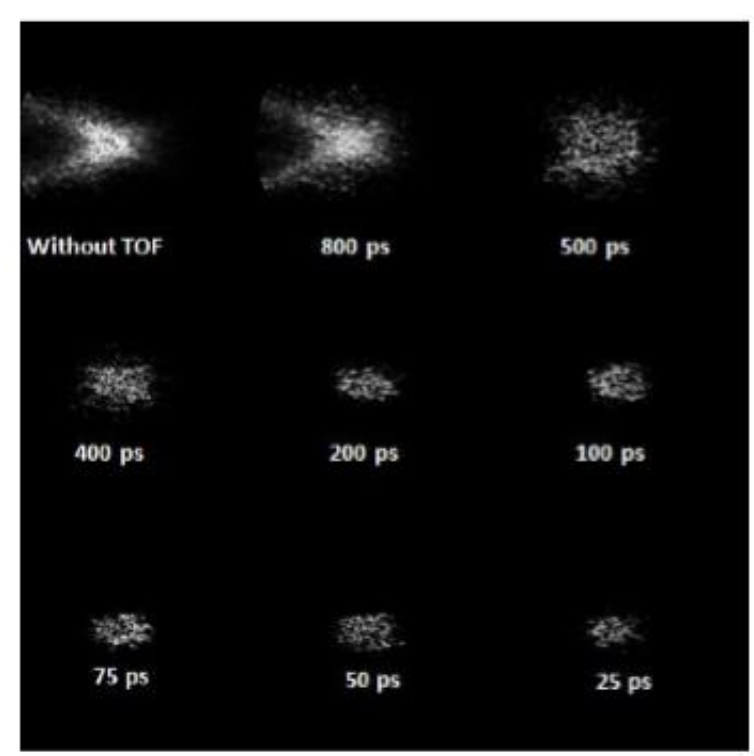

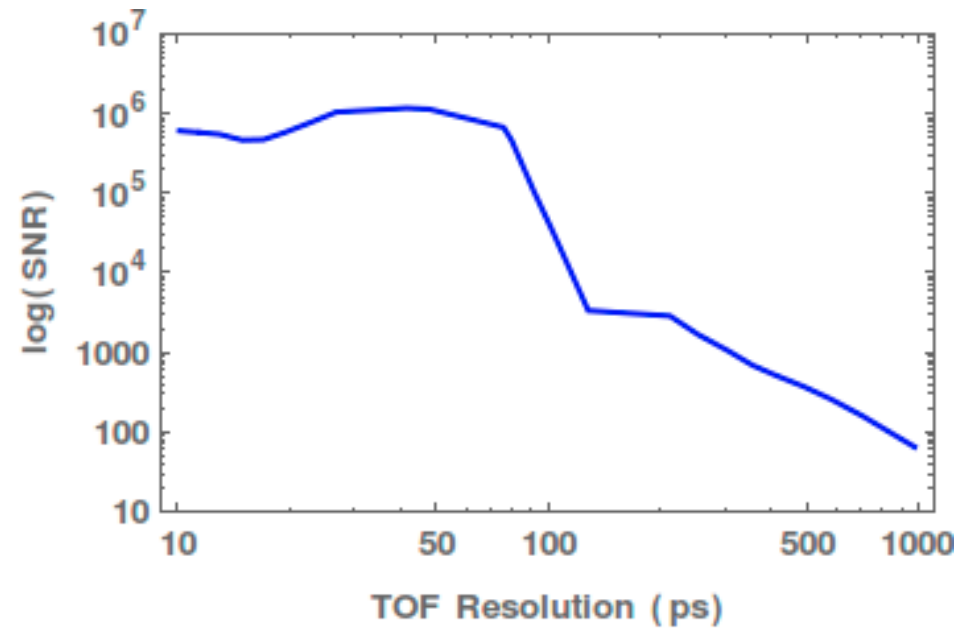

Figure 7. Left: reconstructed simulated cylinder for PROSPET geometry with different TOF resolutions. Right: logarithm of the SNR vs TOF resolution.

\section{References}

[1] S. R. Cherry, T. Jones, J. S. Karp, J. Qi, W. Moses, and R. Badawi, "Total-Body Pet: Maximizing Sensitivity To Create New Opportunities for Clinical Research and Patient Care," J. Nucl. Med., p. jnumed.116.184028, 2017.

[2] A. J. Gonzalez, F. Sanchez, and J. M. Benlloch, "Organ-Dedicated Molecular Imaging Systems,” IEEE Trans. Radiat. Plasma Med. Sci., vol. 7311, no. c, pp. 1-1, 2018.

[3] Takayuki Y. et al. "Development of Pr:LuAG Scintillator Array and Assembly for Positron Emission Mammography" TNS, Vol. 57, 3, 2010

[4] A. M Ahmed, Hideaki Tashima, Eiji Yoshida, Fumihiko Nishikido and Taiga Yamaya, "Simulation study comparing the helmet-chin PET with a cylindrical PET of the same number of detectors" Phys. Med. Biol. 62 (2017) 4541

[5] Wendie A. et al., "High-Resolution Fluorodeoxyglucose Positron Emission Tomography with Compression ("Positron Emission Mammography") is Highly Accurate in Depicting Primary Breast Cancer" The Breast Journal, Volume 12 Number 4, 2006309 323, 2006, DOI: 10.1111/j.1075-122X.2006.00269.x

[6] Taiga Y. et al., "A proposal of an open PET geometry”. Phys. Med. Biol. 53 (2008) 757773

[7] L. Moliner et al., "Implementation and analysis of list mode algorithm using tubes of response on a dedicated brain and breast PET," Nucl. Instruments Methods Phys. Res. Sect. A Accel. Spectrometers, Detect. Assoc. Equip., vol. 702, pp. 129-132, 2013.

[8] A. J. Reader, R. Manavaki, S. Zhao, P. J. Julyan, D. L. Hastings, and J. Zweit, "Accelerated list-mode EM algorithm," IEEE Trans. Nucl. Sci., vol. 49, no. 1 I, pp. 4249, 2002. 
[9] D. L. M. Rebecca A. Hubbard, Karla Kerlikowske, Chris I. Flowers, Bonnie C. Yankaskas, Weiwei Zhu, "Cumulative probability of false-positive recall or biopsy recommendation after 10 years of screening mammography," vol. 155, no. 8, pp. 481492, 2012.

[10] C. H. Michael O'Connor, Deborah Rhodes, "Molecular Breast Imaging | Breastcancer.org," Expert Rev Anticancer Ther., vol. 9, no. 8, pp. 1073-1080, 2008.

[11] L. Moliner et al., "Performance characteristics of the MAMMOCARE PET system based on NEMA standard," J. Instrum., vol. 12, no. 1, 2017.

[12] A. Heidenreich et al., "EAU guidelines on prostate cancer. Part 1: Screening, diagnosis, and treatment of clinically localised disease," Eur. Urol., vol. 59, no. 1, pp. 61-71, 2011.

[13] N. Klemann et al., "Risk of prostate cancer diagnosis and mortality in men with a benign initial transrectal ultrasound-guided biopsy set: a population-based study," Lancet Oncol., vol. 18, no. 2, pp. 221-229, 2017.

[14] A. M. Grant, T. W. Deller, M. M. Khalighi, S. H. Maramraju, G. Delso, and C. S. Levin, "NEMA NU 2-2012 performance studies for the SiPM-based ToF-PET component of the GE SIGNA PET/MR system," Med. Phys., vol. 43, no. 5, pp. 2334-2343, 2016.

[15] M. Ito, M. S. Lee, and J. S. Lee, "Continuous depth-of-interaction measurement in a single-layer pixelated crystal array using a single-ended readout.," Phys. Med. Biol., vol. 58, no. 5, pp. 1269-1282, 2013. 